\title{
Clinical Triaging in Cough Clinic Alleviates COVID-19 Overload in Emergency Department in India
}

\author{
Neelabh Nayan ${ }^{1} \cdot$ M Krishna Kumar $^{2} \cdot$ Ranjith K Nair $^{1}$ • Ishita Manral ${ }^{1} \cdot$ Snehasree Ghosh ${ }^{1} \cdot$ Sharad Bhalla $^{3}$. \\ Jasdeep Singh $^{3} \cdot$ Anuradha Monga $^{1} \cdot$ Mohd Afzal $^{1} \cdot$ Rajan Kapoor $^{1}$ (I)
}

Accepted: 9 December 2020 / Published online: 7 January 2021

(C) The Author(s), under exclusive licence to Springer Nature Switzerland AG part of Springer Nature 2021

\begin{abstract}
The importance of this study is the efficacy of "symptoms only" approach at a screening clinic for coronavirus disease 2019 (COVID-19) diagnosis in low- and middle-income countries (LMIC) setting. The objective of this study was to assess how efficiently primary care physicians at the screening clinic were able to predict whether a patient had COVID-19 or not, based on their symptom-based assessment alone. The current study is a cross-sectional retrospective observational study. This study was conducted at a single-center, tertiary care setting with a dedicated COVID-19 facility in a metropolitan city in eastern India. Participants are all suspected COVID-19 patients who presented themselves to this center during the outbreak from 1 August 2020 to 30 August 2020. Patients were referred to the Cough Clinic from the various outpatient departments of the hospital or from smaller satellite centers located in different parts of the city and other dependent geographical areas. The main outcome(s) and measure(s) is to study whether outcome of confirmatory test results can be predicted accurately by history taking alone. From 01 August 2020 to 30 Aug 2020, 511 patients with at least one symptom suggestive of COVID-19 reported to screening clinic. Out of these, $65.4 \%$ were males and $34.6 \%$ were females. Median age was 45 years with range being 01 to 92 years. Fever was seen in $70.4 \%$ while cough was present in $22 \%$ of cases. Overall positivity for SARS-CoV-2 during this period in this group was $54.21 \%$. At $50 \%$ pre-test probability, the sensitivity of trained doctors working at the clinic, in predicting positive cases based on symptoms alone, was approximately $74.7 \%$, and specificity for the same was $58.12 \%$. The positive predictive value of the doctors' assessment was $67.87 \%$, and the negative predictive value was $66.02 \%$. Rapid triaging for confirmatory diagnosis of COVID-19 is feasible at screening clinic based on history taking alone by training of primary care physicians. This is particularly relevant in LMIC with scarce healthcare resources to overcome COVID-19 pandemic.
\end{abstract}

Keywords COVID-19 $\cdot$ History taking $\cdot$ Rapid triaging $\cdot$ Symptoms only approach

This article is part of the Topical Collection on Covid-19

Rajan Kapoor

majrkapoor@gmail.com

Neelabh Nayan

neelabh.nayan@gmail.com

M Krishna Kumar

krishnakm312@gmail.com

Ranjith K Nair

ranjith3246@gmail.com

Ishita Manral

atihsi532@gmail.com

Sharad Bhalla

bhallasharad@gmail.com
Jasdeep Singh

jasdeep1077@hotmail.com

Anuradha Monga

dranurahamonga@yahoo.com

Mohd Afzal

afzalmohd4@gmail.com

1 Department of Medicine, Command Hospital (Eastern Command), Alipore Road, Kolkata, West Bengal 700027, India

2 Department of Anesthesia \& Critical Care, Command Hospital, Kolkata, India

3 Department of Pathology, Command Hospital, Kolkata, India 


\section{Introduction}

The outbreak of coronavirus disease 2019 (COVID-19) has ensnared the world in one of the most crippling pandemics in recent times. Despite concerted efforts, India had clocked the world's fastest growing outbreak of COVID-19 in absolute numbers [1]; and the impact of this pandemic has been devastating, especially in low- and middle-income countries (LMIC) like India. In this scenario, it becomes imperative to adopt management strategies that would enable healthcare workers (HCW) to ensure effective triaging of COVID-19 patients to ensure early diagnosis and rapid initiation of treatment for affected patients. Redesigning clinical workflows, reorganizing outpatient settings, and continuous training of primary care physicians to improve pre-test history and symptom-based predictive accuracy of identifying potential cases are some strategies that can mitigate the risk of exposure to CoVID-19 among healthcare workers and other individuals visiting the hospital.

Ours is a 1000-bedded tertiary care hospital in eastern India, which had to modify its practice at the onset of the COVID-19 pandemic. Besides the creation of a 100-bedded exclusive facility for in-patient management of severe COVID-19 patients, a "Cough Clinic" was made on the lines of "fever clinics," the concept of which has grown with the course of the COVID-19 pandemic.

\section{Materials and Methods}

\section{Study Design and Participants}

For this single-center, cross-sectional, retrospective, observational study, all symptomatic patients visiting a specially designated area, the "Cough Clinic" of a Tertiary Hospital in southern part of West Bengal, India, were included. All symptomatic patients who reported to the "Cough Clinic" of the hospital were studied. Asymptomatic contacts as well as patients undergoing COVID-19 testing as part of screening have not been included.

\section{Methodology}

This study was carried out in a tertiary care multi-specialty hospital, which has a dedicated wing for COVID-19 patients since the beginning of March 2020. The current study is a cross-sectional retrospective observational study of presenting symptoms of suspected COVID-19 patients who presented themselves to this center during the outbreak of 2019-2020 from 1 August 2020 to 30 August 2020. It also evaluates the pre-test history and symptom-based predictive accuracy of primary care physicians in identifying potentially positive cases. As this is an observational non-interventional study with no invasion of patient privacy, ethical clearance has been dispensed with.

The "Cough Clinic" was a standalone double-storied building, adjacent to the Emergency Department of the hospital, which initially served as a place to receive and stock medical stores prior to their dispatch to the various wards and departments. It was converted into a designated place to receive, register, and render initial care to any patient with symptoms indicative of COVID-19 infection. Any patient coming to hospital with complaints of fever or respiratory tract involvement such as shortness of breath and cough is referred to the Cough Clinic. Patients were referred to the Cough Clinic from the various outpatient departments of the hospital or from smaller satellite centers located in different parts of the city and other dependent geographical areas.

\section{Design and Layout}

The Cough Clinic, a two-storied structure, is located at a linear distance of approximately $200 \mathrm{~m}$ from the main entrance to the hospital. The entrance to the clinic is by foot or ambulance through an approach road that had been cordoned off and made off-limits to everyone else. The ground floor of the clinic serves as the main area for receiving and stabilizing critical patients, assessing the stable patients, for drawing blood samples, and taking chest radiograph wherever indicated. The room has three emergency beds which are spaced at a distance of $2 \mathrm{~m}$ from each other, and two tables at the far corners with a distance of more than $10 \mathrm{~m}$ between them. The first floor of the clinic serves as the main room where naso-pharyngeal and oro-pharyngeal swabs are drawn for RT-PCR testing for severe acute respiratory syndrome coronavirus-2 (SARS-CoV-2) and stored as per established guidelines [2].

\section{Resource Management and Continuous Training of HCW}

A team of six doctors had been earmarked from the beginning of March, and the same team continued to work there in rotation during the working hours of the hospital. Off-duty hours, the center is manned by doctors taken from a central roster, in a sequential order, assisted by other HCW. Three doctors from the day team work for a period of 2 weeks at a time, following which they undergo 2 weeks of institutional quarantine. The off-duty hours doctors worked for one shift of $12 \mathrm{~h}$ each. With each passing day, as the pandemic evolved, our knowledge of the symptoms, danger signals, early therapeutic goals, and management protocols also evolved. Simultaneously, the designated doctors were re-trained at the beginning of each cycle of 4 weeks in recognizing the clinical features of the disease and were brought up to date with the latest relevant 
information about the disease. In addition, the same briefing was conducted for the entire staff manning the clinic in offduty hours.

\section{Clinical Workflow}

The patients referred to the Cough Clinic are directed to the clinic by various means, which include non-contact thermal screening at the entrance to the hospital, a screening for potential symptoms by a HCW at the entrance to the hospital, and large multi-lingual billboards and signage pointing the way. On arrival at the Cough Clinic, the patients who are stable sit in an open area outside the building. The patients who arrive in ambulances, with ongoing oxygen support, or require any other monitoring or supportive therapy like ventilation etc. are received on the beds inside the clinic. The seating area is in the open, with chairs spaced at least $2 \mathrm{~m}$ apart in all directions. The ground floor is manned by at least three doctors during the working hours, and one during the offworking hours. They are assisted by a trained team of six paramedical HCW in each shift that lasts for $8 \mathrm{~h}$. All the personnel are dressed in personal protective equipment (PPE) at all times during their duty. The patients are called inside the clinic serially, with no more than two patients allowed inside the clinic at one time, and if there is a patient in any of the emergency beds, then no patients are called inside at all.

\section{Medical History and Symptom-Based Pre-test Probability Assessment for COVID-19 and Triaging}

After taking detailed history, including history of travel and contact with suspected or confirmed cases of COVID-19, and presence or absence of comorbidities, the patients are examined. At this point of time, the attending doctor notes down presence or absence of fever and one more important symptom for which the patient has reported to the Cough Clinic. The doctor then makes a pre-test probability of the patient to be actually suffering from COVID-19 which is conveyed telephonically to the consultant who is the overall in-charge of the care of all patients with COVID-19 and makes a final decision regarding the disposal of each patient.

\section{Diagnostic Testing}

After documentation of the patient's particulars, symptoms, and pre-test probability of a positive RT-PCR test based on the doctor's assessment, eligible patients undergo further investigations which include sampling of blood for hematology and biochemistry, radiography of the chest, and filling up of the form for sampling for assessing SARS-CoV-2 infection by real-time polymerase chain reaction (RT-PCR) $[3,4]$.

\section{Post-test Triaging and Patient Management}

Those patients who are deemed stable or at a low risk for SARS-CoV-2 infection are sent home for isolation, and those who are unstable or at a high risk for the infection are placed in the containment rooms in a staging ward pending confirmation of the infection. The reports of all samples collected during the working hours are made available in the late evening hours following which appropriate action is taken at the ward to shift the positive cases to the COVID ward. Those patients who were sent home and are detected to be positive are called back to the hospital for admission, along with their household contacts that undergo sampling by naso-pharyngeal and oropharyngeal swab collection for detection of SARS-CoV-2 infection by RT-PCR. Triaging and management is done as per institutional protocols based on available literature and guidelines $[5,6]$.

The objective of this study was to assess how efficiently primary care physicians at the "Cough Clinic" were able to predict whether a patient had COVID-19 or not, based on their clinical assessment alone. They were asked to ascribe a pretest probability of the patient having COVID-19, based on their assessment in the Cough Clinic. This data was entered and later on compared with the actual result based on RT-PCR testing.

Statistical analysis and data visualization have been done in statistical programming language $\mathrm{R}$ version 3.6.3. Receiver operating characteristics (ROC) curves were used to summarize the accuracy of predictions based on presenting symptoms.

\section{Results}

We present our findings of 1 month from the Cough Clinic (1 August 2020 to 30 August 2020). During this time, we encountered a total of 511 patients with at least one symptom at the clinic, with an average of 16.48 patients per day. Median age was 45 years ranging from 1 to 92 years (Fig. 1). Among these, $334(65.36 \%)$ were males and 177 (34.64\%) were females. Fever was the commonest presenting symptom, which was present in 360 (70.4\%) patients. One hundred two (20\%) patients had only fever without any other symptoms. Other major presenting symptoms were cough (22\%), shortness of breath $(15 \%)$, and body aches $(11 \%)$.

The total number of patients from the Cough Clinic who were found to be positive was $277 / 511$ with overall positivity rate of $54.21 \%$ (Fig. 2). There was no significant difference in positivity rates according to gender of the patients (Fig. 3).

We tried to analyze presenting symptoms, other than fever, according to final RT-PCR positivity for COVID-19. Among the various symptoms, the highest positivity rates were for 


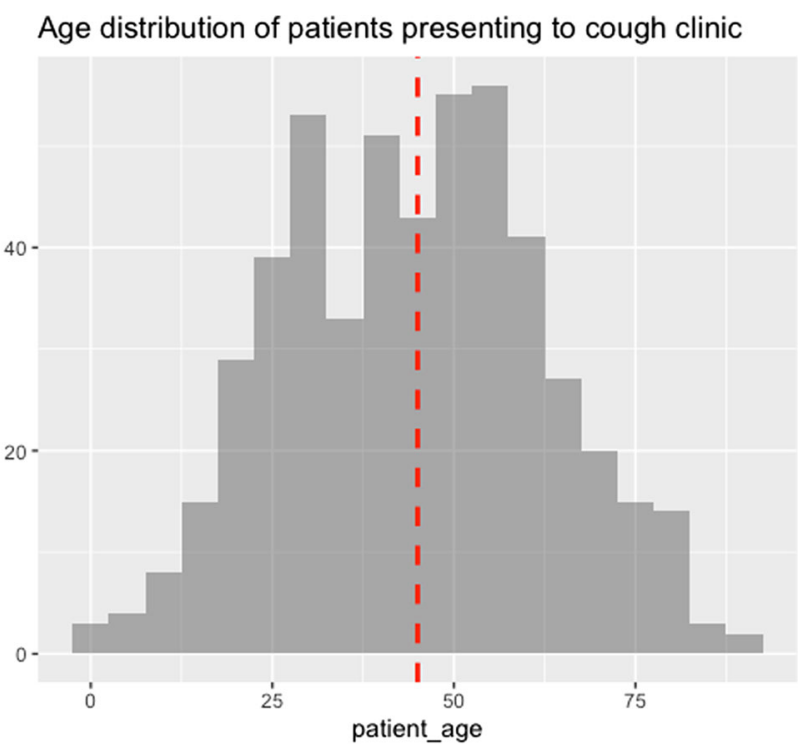

Fig. 1 Histogram depicting age distribution of patients presenting to "Cough Clinic." Median age of 45 years is shown as red line

anosmia (23/30, 76.67\% positivity) and dyspnea (46 out of 74 positives, $62.16 \%$ positivity), whereas the lowest positivity was for rash, where only 2 out of 7 were positive (28.57\%). Table 1 depicts the distribution and positivity rates of the different presenting symptoms of all the patients who presented to the clinic.

We devised a method to assess how efficiently our doctors were making the call whether a patient had COVID-19 or not, based on their clinical assessment alone. They were asked to ascribe a pre-test probability of the patient having COVID-19, based on their assessment in the Cough Clinic. This data was entered and later on was compared with the actual result based on RT-PCR testing. Confusion matrix for predicted versus actual outcome was made which is depicted in Fig. 4.

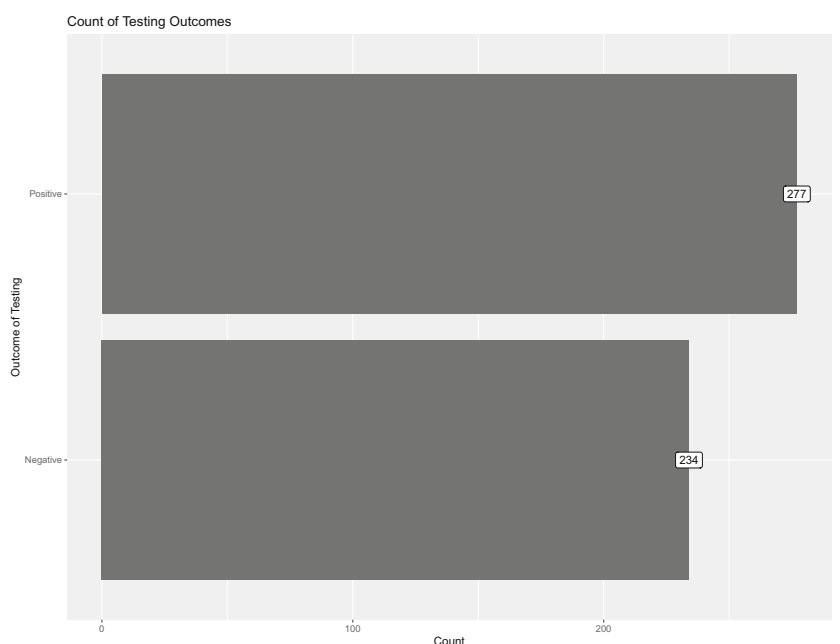

Fig. 2 Bar plots showing final PCR results of nasal and nasopharygeal swabs of patients presenting at Cough Clinic

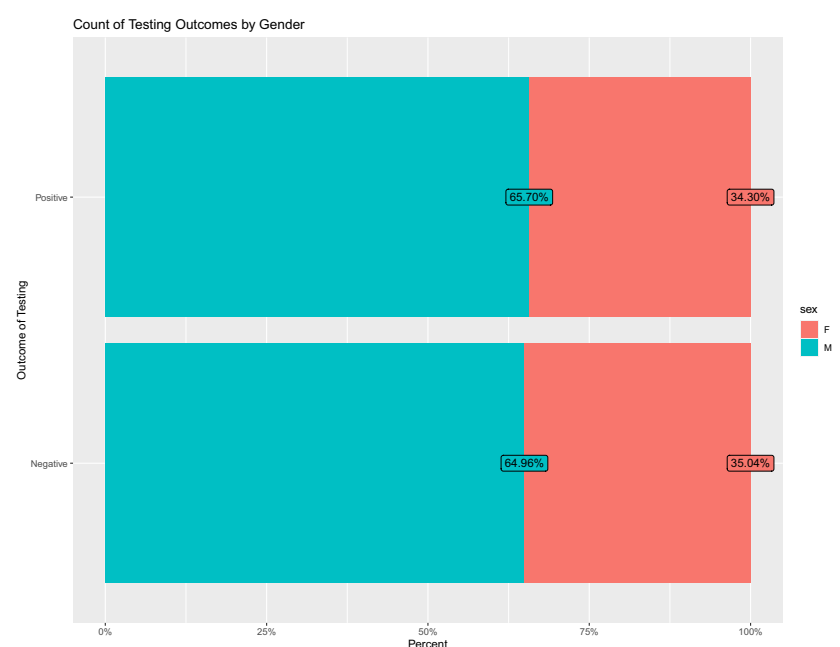

Fig. 3 Outcome of PCR testing stratified according to gender. Bar plots show similar rates of positivity in males and females

To assess the efficacy of doctors involved in triaging patients at the Cough Clinic, receiver operating characteristic (ROC) curve was plotted (Fig. 5) At 50\% pre-test probability, the sensitivity of trained and re-trained doctors working at the clinic, in predicting positive cases based on clinical assessment alone, was approximately $74.7 \%$, and specificity for the same was $58.12 \%$. The positive predictive value of the doctors' assessment was $67.87 \%$, and the negative predictive value was $66.02 \%$.

\section{Discussion}

The SARS-CoV-2 infection brought nations to a grinding halt since its global spread in February 2020. Even though India took early and energetic steps to reduce the transmission of the virus, while working to augment the already over-burdened state and private healthcare systems and beefing up the

Table 1 Presenting symptoms and their positivity rates among all cases presenting to the clinic

\begin{tabular}{lllll}
\hline Presenting symptom & Total & Positive & Negative & Positive \% \\
\hline Cough & 111 & 54 & 57 & 48.65 \\
Nil & 102 & 60 & 42 & 58.82 \\
Dyspnea & 74 & 46 & 28 & 62.16 \\
Body ache & 58 & 27 & 31 & 46.55 \\
Sore throat & 45 & 22 & 23 & 48.89 \\
Anosmia & 30 & 23 & 7 & 76.67 \\
Headache & 28 & 15 & 13 & 53.57 \\
Fatigue & 20 & 8 & 12 & 40 \\
Coryza & 19 & 11 & 8 & 57.89 \\
Diarrhea & 17 & 9 & 8 & 52.94 \\
Rash & 7 & 2 & 5 & 28.57 \\
\hline
\end{tabular}




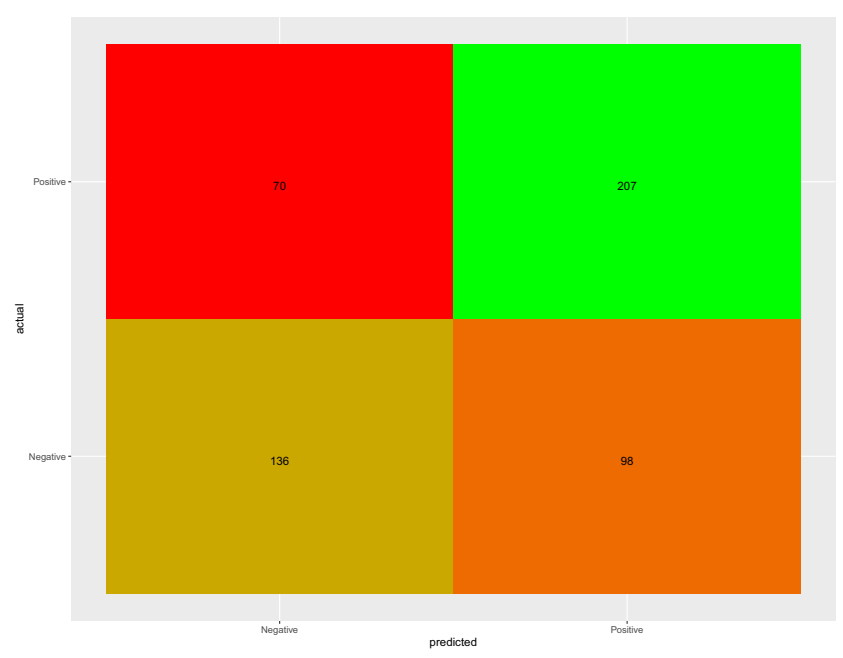

Fig. 4 Confusion matrix of predicted vs actual cases of COVID-19. Positive in Predicted (x-axis) is when assessing doctors reported pre-test probability of more than $50 \%$. Actual (y-axis) is final diagnosis of COVID-19 by RT PCR done on nasal and nasopharyngeal swab

production of personal protective equipment (PPE) and medical supplies and equipment for the disease, the pandemic continues to rage unabated. With no definite treatment till date, the only proven strategy is rapid triaging of patients reporting to hospitals with suggestive symptoms and subjecting patients with high probability to confirmatory tests and subsequent disposal based on test results and their clinical condition [6]. In a populous country like India where hospitals are already overcrowded, there is a need to develop "symptoms only" strategy for initial rapid triaging at any fever or

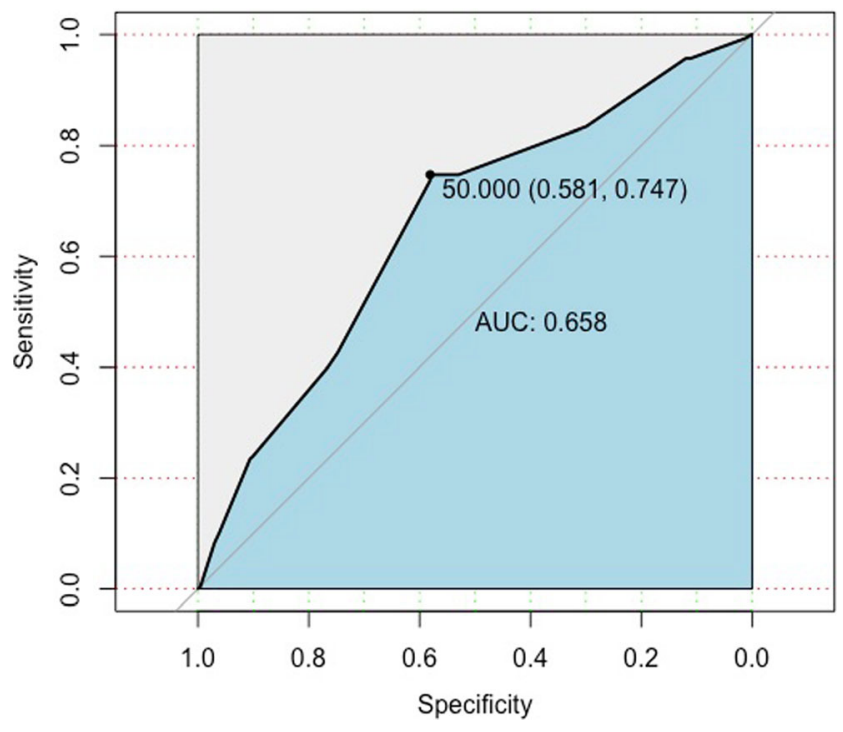

Fig. 5 Receiver operating characteristic (ROC) curve of major symptoms as predictor of COVID-19 positivity by PCR done on nasal and nasopharyngeal swabs. At pre-test probability of $50 \%$, this model has sensitivity of $74.7 \%$ and specificity of $58.1 \%$ cough clinic and leaving detailed physical examination, imaging, and lab tests for the next level, i.e., wards.

Our RT-PCR test positivity rate in symptomatic patients was $54.21 \%$. The overall national positivity for COVID-19 tests conducted during this period was $8.44 \%$ [7]. The national testing methods have only recently allowed testing on demand, and this figure shows that a good screening method can yield a high yield of positivity figures.

Among the symptoms with which patients of COVID-19 presented, we found that the fever was most common one $(70.4 \%)$ followed by cough $(22 \%)$. In a study from China, fever was present in $98 \%$ of patients [8]. In another study from India, the incidence of fever was very low (38.3\%) [9]. Assessment of symptom positivity rates revealed that anosmia yielded a $76.67 \%$ positivity rate for COVID-19, followed by dyspnea at $62.16 \%$. Our work contrasts with that of Hornuss et al. [10], who studied 45 consecutive patients with COVID19 in early May 2020 and found that $40 \%$ of them were suffering from anosmia. However, studies by Vaira et al. suggest a figure of $73.6 \%$, closer to our findings [11, 12]. Based on this aspect of the symptomatology, we suggest further research to include this important symptom as a part of screening procedures in contacts undergoing isolation.

Another interesting aspect to emerge from our study was the result of training of doctors working in the "Cough Clinic." The same team, working seamlessly at the same site, with regular training and update of knowledge was able to predict the presence of infection with confidence in $74.73 \%$ cases on clinical basis alone. With reinforcement and repeated exposure to the clinical scenario, this figure can only go up. It will be a big boost to healthcare settings elsewhere if similar models can replicate this positivity rate. Trained $\mathrm{HCW}$ can detect cases of COVID-19 in the OPD with reasonable certainty, and then direct appropriate allocation of testing and isolation-quarantine measures, thus leading to efficient allocation of the already limited resources. Moreover, at initial review, triaging by symptoms alone is not only fast but also decreases the overall risk of transmission of infection to the $\mathrm{HCW}$ if they were to conduct detailed physical examination. To our knowledge, this is the first study where triaging has been done based purely on history taking and symptomatology.

Our model can be replicated and then modified upon by other multi-specialty centers to ensure that healthcare providers do not suffer during this pandemic while ensuring that patients with COVID-19 are physically isolated early on, so as to allow all other aspects of a multi-specialty hospital to function. The model can be further integrated with telehealth approaches like phone screening, mobile applications, online screening tools, or videoconferencing/ telemedicine visits to screen patients for symptoms of COVID-19 and decide whether the patient is required to visit a medical facility for further evaluation and management. 
Authors' Contributions All the listed authors have made substantial contributions to the conception and the design of the study and the acquisition, analysis, or interpretation of sections of data for the work. All the listed authors have been involved in drafting the paper and revising it critically for important intellectual content, and all were involved in the final approval of the version to be published. All the authors agree to be held accountable for all aspects of the work in ensuring that questions related to the accuracy or integrity of any part of the work are appropriately investigated and resolved.

Data Availability All relevant data and material is available with the corresponding author.

\section{Compliance with Ethical Standards}

Conflict of Interest The authors declare that they have no conflict of interest.

Ethics Approval Ethics approval was waived off, in view of the ongoing pandemic and relevance of the study in mitigating the same. However, all procedures performed in studies involving human participants were in accordance with the ethical standards of the institutional and/or national research committee and with the 1964 Helsinki declaration and its later amendments or comparable ethical standards.

Consent to Participate All the participants in the study were included after provision of informed written consent.

Consent for Publication Not applicable.

Code Availability Not applicable.

\section{References}

1. Worldometers info Available from: https://www.worldometers. info/coronavirus/. Accessed 1 Oct 2020.

2. Cdc.gov. Available from: https://www.cdc.gov/coronavirus/2019ncov/lab/guidelines-clinical-specimens.html. Accessed 1 Oct 2020.
3. Wang W, Xu Y, Gao R, Lu R, Han K, Wu G, et al. Detection of SARS-CoV-2 in different types of clinical specimens. JAMA. 2020. https://doi.org/10.1001/jama.2020.3786.

4. Pfefferle S, Reucher S, Norz D, Lutgehetmann M. Evaluation of a quantitative RT-PCR assay for the detection of the emerging coronavirus SARS-CoV-2 using a high throughput system. Euro Surveill. 2020. https://doi.org/10.2807/1560-7917.Es.2020.25.9. 2000152.

5. World Health Organization. Clinical management of severe acute respiratory infection when novel coronavirus $(\mathrm{nCoV})$ infection is suspected. Interim guidance, World Health Organization (WHO). 2020. https://www.who.int/publications-detail/ clinicalmanagement-of-severe-acute-respiratory-infection-whennovelcoronavirus-(ncov)-infection-is-suspected. Accessed 3 Oct 2020 .

6. Zhang J, Zhou L, Yang Y, Peng W, Wang W, Chen X. Therapeutic and triage strategies for 2019 novel coronavirus disease in fever clinics. Lancet Respir Med. 2020;8(3):e11-2. https://doi.org/10. 1016/S2213-2600(20)30071-0.

7. COVID-19 India resource centre. Available from: http:// covidindiaupdates.in. Accessed 3 Oct 2020.

8. Huang C, Wang Y, Li X, Ren L, Zhao J, Hu Y, et al. Clinical features of patients infected with 2019 novel coronavirus in Wuhan, China. Lancet. 2020;395(10233):497-506.

9. Dosi R, Jain G, Mehta A. Clinical characteristics, comorbidities, and outcome among 365 patients of coronavirus disease 2019 at a tertiary care centre in central India. J Assoc Physicians India. 2020;68:14-9.

10. Hornuss D, Lange B, Schröter N, Rieg S, Kern WV, Wagner D. Anosmia in COVID-19 patients. Clin Microbiol Infect. 2020;26(10):1426-7.

11. Vaira LA, Deiana G, Fois AG, Pirina P, Madeddu G, De Vito A, et al. Objective evaluation of anosmia and ageusia in COVID-19 patients: single-center experience on 72 cases. Head Neck. 2020;42(6):1252-8.

12. Vaira LA, Salzano G, Petrocelli M, Deiana G, Salzano FA, De Riu G. Validation of a self-administered olfactory and gustatory test for the remotely evaluation of COVID-19 patients in home quarantine. Head Neck. 2020;42(7):1570-6.

Publisher's Note Springer Nature remains neutral with regard to jurisdictional claims in published maps and institutional affiliations. 